Research Article

www.jestr.org

\title{
Research on the Measurement Error of MWIR Average Atmospheric Transmittance
}

\author{
Yuanyuan Ji, Wenhai Xu", Ying Li, Qilei Cao, Yukun Sun, Debin Ma and Ming Zhao \\ College of Information Science Technology of Dalian Maritime University, Dalian, Liaoning province, 116026, China
}

Received 16 August 2013; Accepted 17 January 2014

\begin{abstract}
The atmospheric transmittance in mid-wavelength infrared (MWIR) band reflects the characteristics of atmospheric particles, which is typically used in MWIR imaging system correction and data extraction. MWIR imager is wide spectral band instrument and its measuring output is usually affected by broadband atmospheric transmittance which is usually considered as average influence, therefore it is exactly of great importance to study the measurement of MWIR average atmospheric transmittance, which is valuable for MWIR images correction and application. A measurement error model of MWIR atmospheric transmittance was proposed according to the measuring method of the broadband average atmospheric transmittance. Because the transmittance measurement principle mentioned in this paper is indirect measurement, some direct input data are needed to be acquired through infrared imager, blackbody, etc. Finally, combining the measurement error theory with data of the experiments, the MWIR atmospheric transmittance and its measurement error are extracted. The results show that the MWIR atmospheric transmittance measured value is reasonable according to the empirical value in sunny day. And the measurement error objectively reflects most aspects of the test, exactly proving the validity of the measurement experiment.
\end{abstract}

Keywords: MWIR average atmospheric transmittance, MWIR imaging system, Measurement error

\section{Introduction}

MWIR average atmospheric transmittance refer to the effects caused by atmospheric molecular and aerosol particles on the radiation transfer of the ground objects, which is an important research content in the field of infrared imaging, road monitoring and city security etc. MWIR broadband average atmospheric transmittance is generally used for correcting the radiation or temperature measurement of infrared scene [1], [2], so that the ground can be observed even in the smog-weary city. Besides, they could be used for infrared imaging generation, simulation [3] and so on, which are of significant value in infrared developments and applications.

There are many measurement methods to get the atmospheric transmission characteristic in the whole world. For instance, Jun Zhu [4], Heli Wei [5] from China presented the measurement methods by using interferometric infrared spectral radiometer to obtain the atmospheric spectral transmittance data. And D. Sadot [6] from Israel suggested using $\mathrm{CO}_{2}$ laser system to measure $10.6 \mu \mathrm{m}$ wavelength atmospheric transmission. Moreover, Michael Engel [7] presented a remote measurement by using a thermal imager to study the transmittance in the interested spectral band.

Compared with the spectral transmittance measurement mentioned above, the research of MWIR wavelength, generally considered as $3 \sim 5 \mu \mathrm{m}$, average atmospheric transmittance has rarely been reported. However, the MWIR average atmospheric transmittance plays an important role in infrared field for they could be used as correction data for MWIR infrared imager, which makes them attractive to the researchers gradually. Ciyin Yang once did some research on the MWIR atmospheric transmittance measure [8], while the measurement error was understated which needed to be paid enough attention in fact to ensure the validity of measurement method and results. Moreover, the affection of spectral response did not been taken into account for the measurement as well as the error source of uncertainty of spectral responsivity, etc.

This paper aims at developing a thorough measurement error theory for the MWIR average atmospheric transmittance. A measurement error model of average transmittance is presented based on the error analysis theory [9]. The error sources from most measure aspects are considered in detail, which are usually neglected by the predecessors. Finally, the MWIR average atmospheric transmittance measurement method and the error are analyzed through the experiment in sunny day, so that the correctness of the measurement method and error evaluation theory is tested according to the experimental data.

\footnotetext{
*E-mail address: whxu@dlmu.edu.cn

ISSN: 1791-2377 @ 2014 Kavala Institute of Technology. All rights reserved.
} 


\section{MWIR average atmospheric transmittance Measurement}

The transmittance should be carried out under a proper method with a special system. Through the error theory, the error can be obtained to prove the validity of the measurement.

\subsection{MWIR average atmospheric transmittance measurement system}

For getting the MWIR average atmospheric transmittance, a measure system is needed. The measurement system includes three parts: the MWIR imaging system, big area blackbody source and a portable computer. MWIR imaging system is an important device for studying the infrared field, which objectively reflects the infrared material (including solids and gases, etc.) characteristics. In this paper, the infrared camera, with the wide spectral of $3 \sim 5 \mu \mathrm{m}$, is selected as measurement instrument. Besides, blackbody source is another significant component tool for measuring the MWIR average atmospheric transmission characteristic. The atmospheric transmittance is measured by adjusting the temperature of the standard blackbody. And the wearable computer is used for record the measured data.

And the schematic diagram of the system test scenario is listed in Fig. 1, which illustrates the measure instrument and their setting position when the system works.

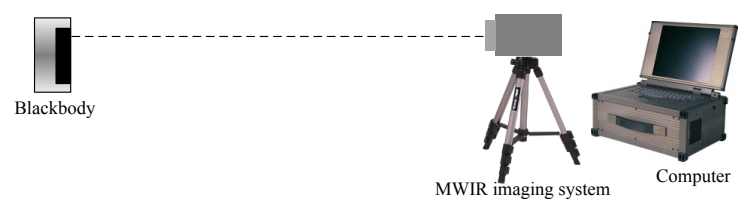

Fig. 1. Atmospheric transmittance measurement scene

The MWIR average atmospheric transmittance of the transmission path between the blackbody target and the infrared imager, indicated by the dashed line, can be measured by the system. Then the experimental output would be stored by the computer.

\subsection{MWIR average atmospheric transmittance measurement principle}

According to theory of the infrared imaging link, the high temperature and low temperature blackbody gray values are expressed by the following formulas:

$$
\begin{aligned}
& g_{H}=K\left(\tau \cdot L_{H}+L_{p a t h}\right)+B \\
& g_{L}=K\left(\tau \cdot L_{L}+L_{p a t h}\right)+B
\end{aligned}
$$

MWIR average atmospheric transmittance can be obtained by simultaneous equations (1) and (2).

$$
\tau=\frac{g_{H}-g_{L}}{K\left(L_{H}-L_{L}\right)}
$$

where $\tau$ is the MWIR average atmospheric transmittance, $g_{H}$ is high temperature blackbody gray value, $g_{L}$ is cryogenic blackbody gray value, $L_{H}$ is infrared camera pupil radiance in zero distance range from the high temperature blackbody, $L_{L}$ is the pupil radiance from the low temperature blackbody and $K$ is the thermal imager radiometric calibration coefficient. $L_{H}$ and $L_{L}$ can be derived from the blackbody parameters and infrared camera's characteristics. According to infrared response rule [10], the following formula can be derived.

$$
L_{H}=\int_{\lambda_{1}}^{\lambda_{2}} \varepsilon(\lambda) \cdot R(\lambda) L_{B H}(\lambda) d \lambda
$$

where $\lambda$ spectral wavelength, $\lambda_{1}=3 \mu \mathrm{m} . \quad \lambda_{2}=5 \mu \mathrm{m} . \varepsilon(\lambda)$ is emissivity of blackbody, $R(\lambda)$ is spectral responsivity of infrared camera and $L_{B H}(\lambda)$ is the spectral radiance of ideal high temperature blackbody, which can be expressed by the following formula according to Planck's blackbody law [11].

$$
L_{B H}(\lambda)=\frac{c_{1}}{\pi \cdot \lambda^{5}\left(e^{\frac{c_{2}}{\lambda T_{H}}}-1\right)}
$$

where $c_{1}$ and $c_{2}$ are the radiation constants, $c_{1}=3.7418 \times 10^{4}$ $\mathrm{W} \cdot \mathrm{cm}^{-2} \mu \mathrm{m}^{4}, \quad c_{2}=1.4388 \times 10^{4} \mu \mathrm{m} \mathrm{K} . \quad T_{H}$ is the surface temperature of the high temperature blackbody. $\pi$ is constant. Similar to $L_{H}, L_{L}$ can be calculated by the following formula.

$$
L_{L}=\frac{1}{\pi} \int_{\lambda_{1}}^{\lambda_{2}} \frac{c_{1} \cdot \varepsilon(\lambda) \cdot R(\lambda)}{\lambda^{5}\left(e^{\frac{c_{2}}{\lambda T_{L}}}-1\right)} d \lambda
$$

Combining Eqs.(1) (5) and considering the spectral characteristics of responsivity and radiation, the broadband average transmittance $\tau$ can be represented by the equation listed below.

$$
\tau=\frac{g_{H}-g_{L}}{K\left(\frac{1}{\pi} \int_{\lambda_{1}}^{\lambda_{2}} \frac{c_{1} \cdot \varepsilon(\lambda) \cdot R(\lambda)}{\lambda^{5}\left(e^{\frac{c_{2}}{\lambda T_{H}}}-1\right)} d \lambda-\frac{1}{\pi} \int_{\lambda_{1}}^{\lambda_{2}} \frac{c_{1} \cdot \varepsilon(\lambda) \cdot R(\lambda)}{\lambda^{5}\left(e^{\frac{c_{2}}{\lambda T_{L}}}-1\right)} d \lambda\right)}
$$

where the parameters are exactly of the same meaning to the nomenclature appear before.

\subsection{MWIR average atmospheric transmittance measurement error}

From the measurement principle, atmospheric transmittance is an indirect measure value, i.e. the value is a function of some direct measurements. Therefore, the atmospheric transmittance error is a function of each directly measurements error.

The relative standard deviation may well reflect the impact of the error. In this paper, the relative standard deviation is unified to represent the synthesis of systematic errors and random errors. After the system errors are corrected, the measurement process can be considered as only random errors left: $\sigma_{r}\left(x_{1}\right), \sigma_{r}\left(x_{\mathrm{i}}\right), \ldots, \sigma_{r}\left(x_{s}\right)$. And then the system error $\sigma_{r}(y)$ is derived, shown as follows:

$$
\sigma_{r}(y)=\sqrt{\sum_{i=1}^{s}\left(\frac{\partial y}{\partial x_{i}}\right)^{2} \sigma_{r}\left(x_{i}\right)^{2}}
$$


The atmospheric transmittance measurement principle shows that the error sources from most of the measuring link: the infrared image value acquisition, the blackbody temperature and infrared imager calibration, et al. According to measurement error synthesis theoretic Eq.(8), atmospheric transmittance measurement error $\sigma_{r}(\tau)$ can be deduced to the mathematical Eq. (9).

$$
\sigma_{r}(\tau)=\sqrt{\begin{array}{l}
\left(\frac{\partial \tau}{\partial\left(g_{H}\right)}\right)^{2} \sigma_{r}^{2}\left(g_{H}\right)+\left(\frac{\partial \tau}{\partial\left(T_{H}\right)}\right)^{2} \cdot \sigma_{r}^{2}\left(T_{H}\right)+\left(\frac{\partial \tau}{\partial\left(T_{L}\right)}\right)^{2} \sigma_{r}^{2}\left(g_{L}\right)+\left(\frac{\partial \tau}{\partial(K)}\right)^{2}\left(T_{L}\right)+\left(\frac{\partial \tau}{\partial(R)}\right)^{2} \cdot \sigma_{r}^{2}(K) \\
+\left(\frac{\partial \tau}{\partial(\varepsilon)}\right)^{2} \sigma_{r}^{2}(\varepsilon)
\end{array}}
$$

where $\sigma_{r}\left(g_{H}\right), \sigma_{r}\left(g_{L}\right), \sigma_{r}\left(T_{H}\right), \sigma_{r}\left(T_{L}\right), \sigma_{r}(K), \sigma_{r}(R)$ and $\sigma_{r}(\varepsilon)$ are respectively the relative standard deviation of $g_{H}, g_{L}, T_{H}, T_{L}$, $K, R$ and $\varepsilon$.

Substituting the Eq.(7), the formula for calculating the MWIR average atmospheric transmittance, to the Greek letter $\tau$ referred by Eq.(9), the average atmospheric transmittance error model can be obtained as the Eq.(10).

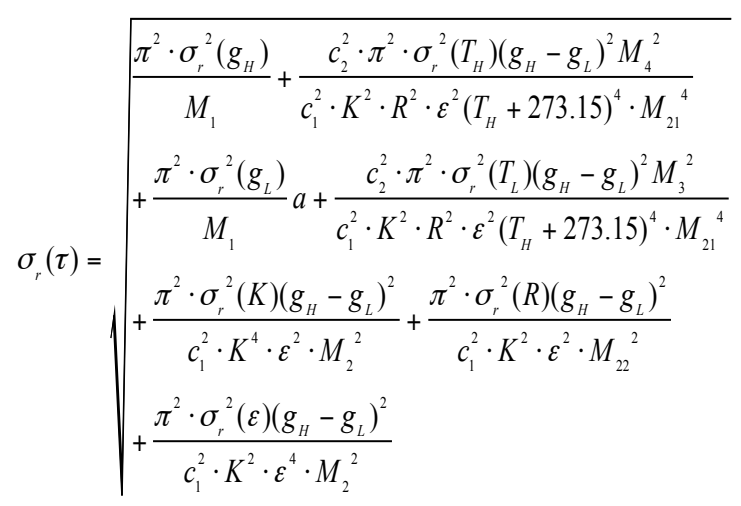

where $M_{1}, M_{2}, M_{21}, M_{22}, M_{3} M_{4}$ is represented by the following integral formulas:

$M_{1}=c_{1}^{2} K^{2} \varepsilon^{2}\left[\int_{\lambda_{1}}^{\lambda_{2}} \frac{R(\lambda)}{\lambda^{5}\left(e^{\lambda\left(c_{H^{2}}+273.15\right)}-1\right)} d \lambda-\int_{\lambda_{1}}^{\lambda_{2}} \frac{R(\lambda)}{\lambda^{5}\left(e^{\lambda\left(c_{2}+273.15\right)}-1\right)} d \lambda\right]^{2}$

$M_{2}=\int_{\lambda_{1}}^{\lambda_{2}} \frac{R(\lambda)}{\lambda^{5}\left(e^{\frac{c_{2}}{\lambda\left(T_{H}+273.15\right)}}-1\right)} d \lambda-\int_{\lambda_{1}}^{\lambda_{2}} \frac{R(\lambda)}{\lambda^{5}\left(e^{\frac{c_{2}}{\lambda\left(T_{L}+273.15\right)}}-1\right)} d \lambda$

$M_{21}=\int_{\lambda_{1}}^{\lambda_{2}} \frac{\sqrt{R(\lambda)}}{\lambda^{5}\left(e^{\frac{c_{2}}{\lambda\left(T_{H}+273.15\right)}}-1\right)} d \lambda-\int_{\lambda_{1}}^{\lambda_{2}} \frac{\sqrt{R(\lambda)}}{\lambda^{5}\left(e^{\frac{c_{2}}{\lambda\left(T_{L}+273.15\right)}}-1\right)} d \lambda$
$M_{22}=\int_{\lambda_{1}}^{\lambda_{2}} \frac{[R(\lambda)]^{2}}{\lambda^{5}\left(e^{\frac{c_{2}}{\lambda\left(T_{H}+273.15\right)}}-1\right)} d \lambda-\int_{\lambda_{1}}^{\lambda_{2}} \frac{[R(\lambda)]^{2}}{\lambda^{5}\left(e^{\frac{c_{2}}{\lambda\left(T_{L}+273.15\right)}}-1\right)} d \lambda$

$M_{3}=\int_{\lambda_{1}}^{\lambda_{2}} \frac{e^{\frac{c_{2}}{\lambda\left(T_{L}+273.15\right)}}}{\lambda^{6}\left(e^{\frac{c_{2}}{\lambda\left(T_{L}+273.15\right)}}-1\right)} d \lambda$

$M_{4}=\int_{\lambda_{1}}^{\lambda_{2}} \frac{e^{\frac{c_{2}}{\lambda\left(T_{H}+273.15\right)}}}{\lambda^{6}\left(e^{\frac{c_{2}}{\lambda\left(T_{H}+273.15\right)}}-1\right)} d \lambda$

where all of symbols have the same meaning to that mentioned before.

The deduced Eq.(10) is exactly the average atmospheric transmittance measurement error model. From formula (10), it can be known that, in order to obtain atmospheric transmittance measurement error, $g_{H}, g_{L}, \sigma_{r}\left(g_{H}\right), \sigma_{r}\left(g_{L}\right), T_{H}, T_{L}$, $\sigma_{r}\left(T_{H}\right), \sigma_{r}\left(T_{L}\right), K, \sigma_{r}(K), R, \sigma_{r}(R), \varepsilon$ and $\sigma_{r}(\varepsilon)$ are needed to be acquired, which are named as input data for getting the measurement value and error of MWIR average atmospheric transmittance.

The acquiring method of these input parameters and their corresponding errors, requires specific measurement trials to support the analysis and calculation. The method, gaining the average atmospheric transmittance by actual measurement with proper measure error, objectively reflects the atmospheric transmittance characteristic of the mid-infrared band, with important value of research and application.

\section{Input data acquisition}

To measure the atmospheric transmittance in terms of Eq.(7), some input parameters, including blackbody temperature, infrared imager gray value and the calibration coefficient, need to be known. This section will introduce the ways of acquiring these input data and the consideration of relative standard deviation.

\subsection{Blackbody temperature acquisition}

Blackbody temperature can be easily collected from the indicating panel. However, it is noteworthy that the blackbody needs around half of an hour to stabilize in a setting temperature. Thus, the temperature acquisition should wait until the blackbody temperature stop floating.

Because the relative standard deviation may well reflect the condition of errors, so the relative standard deviation $\sigma_{r}$ is chosen as unified physical quantity to express the error analyzed in the paper. The errors of blackbody temperature, $T_{H}$ and $T_{L}$, may come from three aspects: Firstly, the inherent error, the difference between the showing value and the actual temperature of the blackbody. Secondly, blackbody surface temperature instability (also called unrepeatability), introducing the testing process with unrepeatability error. Thirdly, the non-uniformity of blackbody, cause the slight 
difference in the temperature blackbody surface area. Thus the error of blackbody temperature can be expressed by Eq.(11) and (12).

$\sigma_{r}\left(T_{H}\right)=\sqrt{\sigma_{\text {in }}^{2}\left(T_{H}\right)+\sigma_{\text {rep }}^{2}\left(T_{H}\right)+\sigma_{u n}^{2}\left(T_{H}\right)}$

$\sigma_{r}\left(T_{L}\right)=\sqrt{\sigma_{\text {in }}^{2}\left(T_{L}\right)+\sigma_{r e p}^{2}\left(T_{L}\right)+\sigma_{u n}^{2}\left(T_{L}\right)}$

where $\sigma_{r}\left(T_{H}\right)$ is relative error of high temperature blackbody, $\sigma_{i n}\left(T_{H}\right)$ is inherent error, $\sigma_{\text {rep }}\left(T_{H}\right)$ is unrepeatable error, $\sigma_{u n}\left(T_{H}\right)$ is the non-uniform error. The meaning of symbols of $T_{L}$ is similar to $T_{H}$.

To test the deviation, the verification experiment is carried out. Due to the blackbody is circular with diameter of $500 \mathrm{~mm}$ rather than a point, five positions in the area are chosen for test, including the middle, up, down, left and right (see Fig. 2).

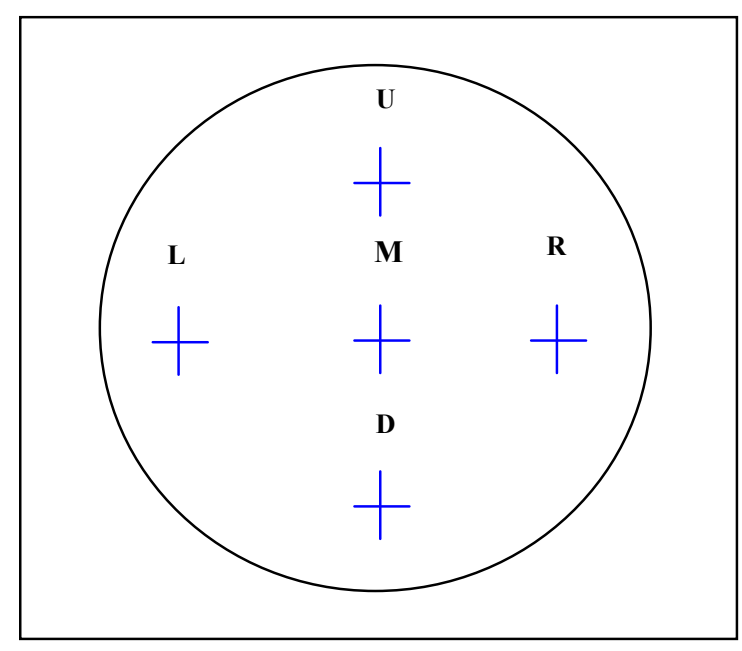

Fig. 2. Test points of blackbody

Considering the far wavelength infrared thermometer as reference instrument, the blackbody temperature can compare with the reference value. Because the nominal value of the emissivity is 0.97 with the error of \pm 0.02 , the emissivity input of FWIR thermometer will be set as $0.95,0.97,0.99$ respectively. In view of the temperature of $15{ }^{\circ} \mathrm{C}$ and $35{ }^{\circ} \mathrm{C}$ will be used for measuring the average transmittance, these two temperature are taken as test values. In addition, there are two options for infrared detection, one is D1, suitable for the range of $0 \sim 125{ }^{\circ} \mathrm{C}$, the other is $\mathrm{D} 2$, suitable for the range of 0 $\sim 500{ }^{\circ} \mathrm{C}$. And D1 is more accurate than D2, so as to choose D1 grade for test. From the test of blackbody, three kinds of errors can be derived, including the inherent error, unrepeatability and non-uniformity. Taking these error source into account, the total error of the temperature of blackbody can be deduced by Eq.(13).
$\sigma_{r}\left(T_{H}\right)=\sqrt{\sigma_{i n}^{2}\left(T_{H}\right)+\sigma_{r e p}^{2}\left(T_{H}\right)+\sigma_{u n}^{2}\left(T_{H}\right)}$

where $\sigma_{r}\left(T_{H}\right)$ is the total error of $T_{H}, \sigma_{i n}\left(T_{H}\right)$ is the inherent error, $\sigma_{r e p}\left(g_{H}\right)$ is the unrepeatable error and $\sigma_{u n}\left(g_{H}\right)$ is the nonuniformity error. And the method of getting the error of $g_{L}$ is the same with $g_{H}$.

\subsection{Infrared images acquisition}

Blackbody can be extracted from the images taken by the MWIR imaging system to get the gray values. To ensure the accuracy of the test, several pictures were taken rather than one, the average of which was considered as the final acquisition result. However, due to the tiny difference between each image, this process would introduce errors.

According to the physical meaning of the relative error, the formula of the error of $g_{H}$ is:

$\sigma_{r}\left(g_{H}\right)=\frac{\sigma\left(g_{H}\right)}{\overline{g_{H}}}$

where $\sigma_{r}\left(g_{H}\right)$ represents the relative standard difference of $g_{H}, \bar{g}_{H}$ represents an average of $g_{H}$, and $\sigma\left(g_{H}\right)$ is the standard deviation that can be estimated from the Bessel formula [12]:

$\sigma\left(g_{H}\right)=\sqrt{\frac{\sum_{i=1}^{n} v_{i}^{2}}{n-1}}$

where $i$ is the number of multiple measurements, $n$ is the measure times, $v_{i}$ is the residual error which formula is shown by Eq.(16).

$v_{i}=g_{H}(i)-\overline{g_{H}}$

where $g_{H}(i)$ is multiple measured values $(i=1,2, \ldots, n)$.

Beyond the unrepeatable error mentioned above, there is another very important error along with the image acquisition, which is the spectral response error. The wide-band infrared radiance is received and converted to gray value by the MWIR imaging system. Due to the uncertainty of spectral response distribution influencing the output data, it is necessary to take the infrared spectral responsivity error into account.

To sum up, the total relative error of image acquisition is a combination of both the unrepeatable error and the spectral response error. In view of these two kinds of error sources, the finalized error can be calculated by Eq.(17).

$\sigma_{r}\left(g_{H}\right)=\sqrt{\sigma_{r e p}^{2}\left(g_{H}\right)+\sigma_{s p e c}^{2}\left(g_{H}\right)}$ 
where $\sigma_{r}\left(g_{H}\right)$ is the total error of $g_{H}, \sigma_{\text {rep }}\left(g_{H}\right)$ is the unrepeatable error and $\sigma_{\text {spec }}\left(g_{H}\right)$ is the spectral responsivity error. And it is the same with the method of getting the error of $g_{L}$.

\subsection{Radiance calibration coefficients aqucisition}

Infrared radiation calibration of the MWIR imaging system is to determine the relationship between the output image and the input radiation energy. Radiometric calibration method is to use a standard blackbody source to analyze the radiometric calibration response coefficient $K$ of the MWIR imager by adjusting the surface temperature of the target. It is remarkable that infrared equipment often have a drift characteristic, which manifests that certain performance parameters of the instrument are alterable along with the time. Thus, shortly before the measure is to be carried out, the infrared device need radiance calibration test to ensure the validity for the measurement.

To calculate the thermal imager radiometric calibration coefficients, the blackbody radiance reaching the entrance pupil of the infrared camera and received by the infrared detector in wide wavelength range under each surface temperature needs to be calculated. And then the data should be fitted to get the radiance calibration coefficient. The fitting model is the linear determined by the linear region characteristic of the infrared imaging system and the application in the measurement. Moreover, the fitting method is least square method, and its meaning is to get the minimum of root mean square error (RMSE).

In addition, due to the uncertainty of the spectral response of infrared imager, the accuracy of the radiometric calibration coefficient can also be affected. Infrared spectral responsivity is described as previously. In consideration of the linear fitting error and infrared spectral error, radiometric calibration coefficients integrated error can be gained by the following expression:

$\sigma_{r}(K)=\sqrt{\sigma_{f i t}^{2}(K)+\sigma_{s p e c}^{2}(K)}$

In the above formula, $\sigma_{r}(K)$ is a comprehensive error of radiance calibration coefficient, $\sigma_{f i t}(K)$ is the fitting error, $\sigma_{\text {spec }}(K)$ is the spectral response error.

\section{Results and discussion}

The measurement of MWIR average atmospheric transmittance was carried out in accordance with the theory and method introduced in section 2 and 3. The experiment was deducted in Dalian when the season is winter. And the weather is nice, atmospheric visibility was $5375 \mathrm{~m}$, the air temperature was $4.6{ }^{\circ} \mathrm{C}$, relative humidity is $45.2 \%$ and the air pressure is $1010.7 \mathrm{hPa}$. The tested distance is $297.1 \mathrm{~m}$, of which the transmittance was measured.

\subsection{Result of Blackbody temperature acquisition}

According to the error test introduced in section 3.1, twice tests were carried out, each of which included at least 10 sets of data, to make sure the results objective and accurate. The data are so long that only the primary parts are listed here. (see Table 1 2).

Table 1. Test of blackbody on $15^{\circ} \mathrm{C}$

\begin{tabular}{|c|c|c|c|c|}
\hline emissivity & point & $\begin{array}{l}\text { test } 1 \\
\left({ }^{\circ} \mathrm{C}\right)\end{array}$ & $\begin{array}{l}\text { test2 } \\
\left({ }^{\circ} \mathrm{C}\right)\end{array}$ & analysis \\
\hline \multirow{5}{*}{0.95} & middle & 15.35 & 15.34 & \multirow{5}{*}{$\begin{array}{l}\text { inherent error }<0.26^{\circ} \mathrm{C} \\
\text { unrepeatability }<0.09^{\circ} \mathrm{C} \\
\text { non-uniformity }<0.06^{\circ} \mathrm{C}\end{array}$} \\
\hline & up & 15.27 & 15.24 & \\
\hline & down & 15.29 & 15.28 & \\
\hline & left & 15.25 & 15.24 & \\
\hline & right & 15.14 & 15.12 & \\
\hline \multirow{5}{*}{0.97} & middle & 14.76 & 14.78 & \multirow{5}{*}{$\begin{array}{l}\text { inherent error }<0.04^{\circ} \mathrm{C} \\
\text { unrepeatability }<0.15^{\circ} \mathrm{C} \\
\text { non-uniformity }<0.13^{\circ} \mathrm{C}\end{array}$} \\
\hline & up & 15.04 & 15.04 & \\
\hline & down & 15.13 & 15.15 & \\
\hline & left & 14.85 & 14.86 & \\
\hline & right & 15.01 & 15.03 & \\
\hline \multirow{5}{*}{0.99} & middle & 15.44 & 15.45 & \multirow{5}{*}{$\begin{array}{l}\text { inherent error }<0.25^{\circ} \mathrm{C} \\
\text { unrepeatability }<0.17^{\circ} \mathrm{C} \\
\text { non-uniformity }<0.13^{\circ} \mathrm{C}\end{array}$} \\
\hline & up & 15.32 & 15.34 & \\
\hline & down & 15.24 & 15.27 & \\
\hline & left & 15.17 & 15.15 & \\
\hline & right & 15.06 & 15.04 & \\
\hline
\end{tabular}

Table 2. Test of blackbody on $35^{\circ} \mathrm{C}$

\begin{tabular}{|c|c|c|c|c|}
\hline emissivity & point & $\begin{array}{l}\text { test } 1 \\
\left({ }^{\circ} \mathrm{C}\right)\end{array}$ & $\begin{array}{l}\text { test2 } \\
\left({ }^{\circ} \mathrm{C}\right)\end{array}$ & analysis \\
\hline \multirow{5}{*}{0.95} & middle & 35.39 & 35.39 & \multirow{5}{*}{$\begin{array}{l}\text { inherent error }<0.27^{\circ} \mathrm{C} \\
\text { unrepeatability }<0.12^{\circ} \mathrm{C} \\
\text { non-uniformity }<0.08^{\circ} \mathrm{C}\end{array}$} \\
\hline & up & 35.26 & 35.24 & \\
\hline & down & 35.31 & 35.32 & \\
\hline & left & 35.28 & 35.29 & \\
\hline & right & 35.1 & 35.08 & \\
\hline \multirow{5}{*}{0.97} & middle & 34.99 & 34.97 & \multirow{5}{*}{$\begin{array}{l}\text { inherent error }<0.07^{\circ} \mathrm{C} \\
\text { unrepeatability }<0.07^{\circ} \mathrm{C} \\
\text { non-uniformity }<0.05^{\circ} \mathrm{C}\end{array}$} \\
\hline & up & 35.12 & 35.15 & \\
\hline & down & 35.1 & 35.11 & \\
\hline & left & 35.06 & 35.05 & \\
\hline & right & 35.07 & 35.07 & \\
\hline \multirow{5}{*}{0.99} & middle & 35.28 & 35.27 & \multirow{5}{*}{$\begin{array}{l}\text { inherent error }<0.25^{\circ} \mathrm{C} \\
\text { unrepeatability }<0.06^{\circ} \mathrm{C} \\
\text { non-uniformity }<0.05^{\circ} \mathrm{C}\end{array}$} \\
\hline & up & 35.29 & 35.28 & \\
\hline & down & 35.30 & 35.28 & \\
\hline & left & 35.23 & 35.19 & \\
\hline & right & 35.18 & 35.17 & \\
\hline
\end{tabular}

From the experiment, it can be concluded three points. First, the measured temperature and the blackbody surface temperature are closest when the FWIR thermometer emissivity is set as 0.97 , while the difference increases with the temperature. And the maximum is about $0.26^{\circ} \mathrm{C} @ 15^{\circ} \mathrm{C}$ and $0.27{ }^{\circ} \mathrm{C} @ 35{ }^{\circ} \mathrm{C}$. Besides, the blackbody has a stable repeatability, less than $0.17^{\circ} \mathrm{C} @ 15^{\circ} \mathrm{C}$ and $0.12{ }^{\circ} \mathrm{C} @ 35^{\circ} \mathrm{C}$. Moreover, the non-uniformity of blackbody source is good, around $0.08{ }^{\circ} \mathrm{C} @ 15^{\circ} \mathrm{C}$ and $0.08^{\circ} \mathrm{C} @ 35^{\circ} \mathrm{C}$.

From Eq.(13) and the input value obtained here, the final error of $T_{H}$ is $0.87 \%$. Similarly, the final error of $T_{L}$ is $2.4 \%$. Obviously, $T_{H}$ is equal to 35 and $T_{L}$ is equal to 15 .

\subsection{Result of Infrared images acquisition}

From section 3.2, the infrared images acquisition experiment was done to record the thermal images of $15{ }^{\circ} \mathrm{C}$ and $35{ }^{\circ} \mathrm{C}$ blackbody, as well as the fluctuation of the grays. The results are shown in Fig.3 and Fig.4. 


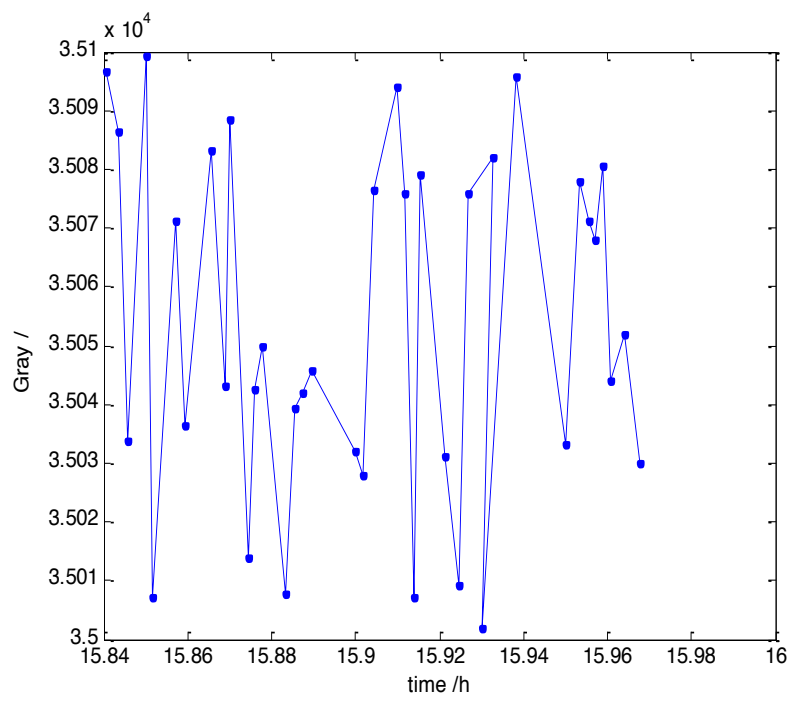

Fig. 3. Gray value of 15 blackbody

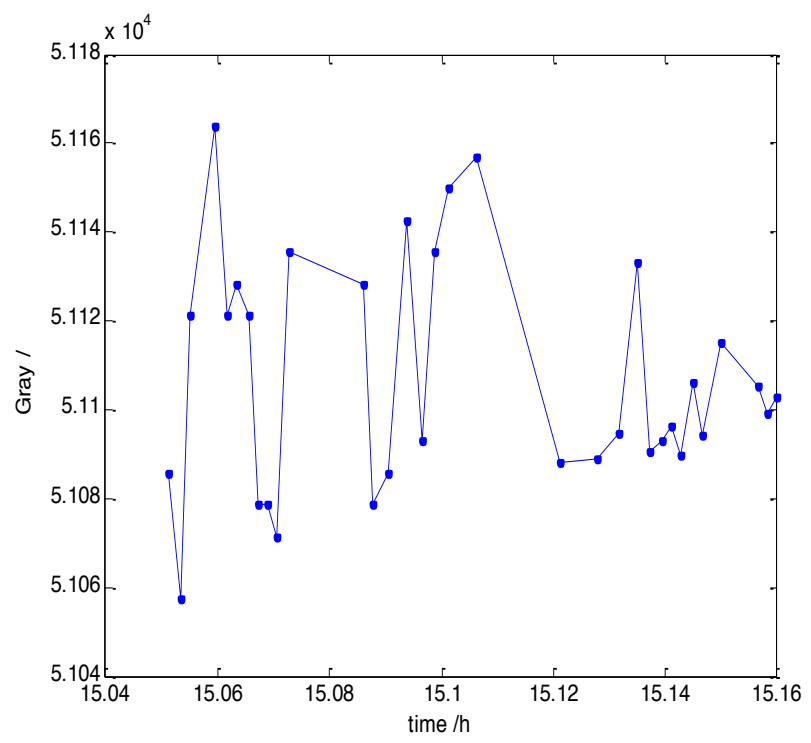

Fig. 4. Gray value of 35 blackbody

Under the data shown by Fig.4 and Eqs.(14) (16), $\sigma_{r}\left(g_{H}\right)$, the error of 35 blackbody can be solved: $\sigma\left(g_{H}\right)=26, \bar{g}$ ${ }_{H}=51107, \sigma_{r}\left(g_{H}\right)=0.05 \%$. Similarly, the error of 15 blackbody $\sigma_{r}\left(g_{L}\right)$ can be obtained: $\sigma\left(g_{L}\right)=26, \bar{g}_{L}=34889, \sigma_{r}\left(g_{L}\right)=0.08 \%$.

Besides of the above error, the spectral response error is another error source. From the manual of infrared detectors, the actual spectral response distribution of MWIR optielectronic detector is more narrow than the nominal cutoff wavelength range of $3 \sim 5 \mu \mathrm{m}$. The related literature Infrared spectral responsivity shows that the spectral response curve can be fitted by cubic spline fitting method, which uses the following formula Eq.(19) and coefficients (see Table 3) for the spectral response characteristic characterization:

$$
R(\lambda)=k_{i 1}\left(\lambda-\lambda_{i}\right)^{3}+k_{i 2}\left(\lambda-\lambda_{i}\right)^{2}+k_{i 3}\left(\lambda-\lambda_{i}\right)+k_{i 4}
$$

where, $\lambda$ is the wavelength, $\lambda_{i}$ is the boundary value of each sub band of the wide wavelength range, $k_{i 1}, k_{i 2}, k_{i 3}, k_{i 4}$ is the cubic spline fitting coefficients.

Table 3. Three times polynomial coefficients

\begin{tabular}{l|c|c|c|c}
\hline \multirow{2}{*}{$\begin{array}{l}\text { Wavelength } \\
\text { range }(\mu \mathrm{m})\end{array}$} & \multicolumn{4}{|c}{ three times polynomial coefficients } \\
\cline { 2 - 5 } & $k_{1}$ & $k_{2}$ & $k_{3}$ & $k_{4}$ \\
\hline $3.50 \sim 3.51$ & -1186.563 & 239.522 & -2.9244 & 0.0000 \\
\hline $3.51 \sim 3.55$ & -1186.563 & 175.913 & 4.4992 & 0.0175 \\
\hline $3.55 \sim 3.59$ & -2115.037 & 48.6935 & 12.5263 & 0.3488 \\
\hline $3.59 \sim 3.63$ & 1519.2942 & -178.07 & 7.9024 & 0.7621 \\
\hline $3.63 \sim 3.70$ & 59.5938 & -12.918 & 0.9818 & 0.8869 \\
\hline $3.70 \sim 3.98$ & 0.0024 & 0.1276 & 0.0486 & 0.9129 \\
\hline $3.98 \sim 4.24$ & -1.5025 & 0.1295 & 0.1207 & 0.9366 \\
\hline $4.24 \sim 4.34$ & 3.3703 & -1.0384 & -0.1148 & 0.9505 \\
\hline $4.34 \sim 4.42$ & 27.0241 & -0.0146 & -0.2214 & 0.9317 \\
\hline $4.42 \sim 4.45$ & -51.3374 & 6.1827 & 0.2501 & 0.9268 \\
\hline \hline & \multicolumn{5}{|c}{} \\
\hline \hline \multirow{2}{*}{ Wavelength } & \multicolumn{5}{|c}{ three times polynomial coefficients } \\
\cline { 2 - 6 } range $(\mu \mathrm{m})$ & $k_{1}$ & $k_{2}$ & $k_{3}$ & $k_{4}$ \\
\hline $4.45 \sim 4.49$ & 4.4862 & 0.6785 & 0.4953 & 0.9413 \\
\hline $4.49 \sim 4.52$ & -60.3999 & 1.1595 & 0.5610 & 0.9600 \\
\hline $4.52 \sim 4.56$ & 42.3133 & -5.3164 & 0.4124 & 0.9788 \\
\hline $4.56 \sim 4.59$ & -368.5867 & -0.7797 & 0.1945 & 0.9887 \\
\hline $4.59 \sim 4.63$ & 717.7669 & -40.298 & -1.274 & 0.9778 \\
\hline $4.63 \sim 4.67$ & -294.7476 & 36.6582 & -1.404 & 0.9136 \\
\hline $4.67 \sim 4.70$ & 475.6533 & 5.0564 & 0.0872 & 0.8968 \\
\hline $4.70 \sim 4.74$ & -2841.599 & 56.0542 & 2.2712 & 0.9281 \\
\hline $4.74 \sim 4.77$ & 1302.2533 & -248.61 & -4.611 & 0.9511 \\
\hline $4.77 \sim 4.80$ & 1302.2533 & -108.99 & -17.39 & 0.5283 \\
\hline \hline
\end{tabular}

Combining the thermal imager chip data with cubic spline fitting method and the fitting coefficients, the infrared spectral response curves can be drawn as Fig. 5.

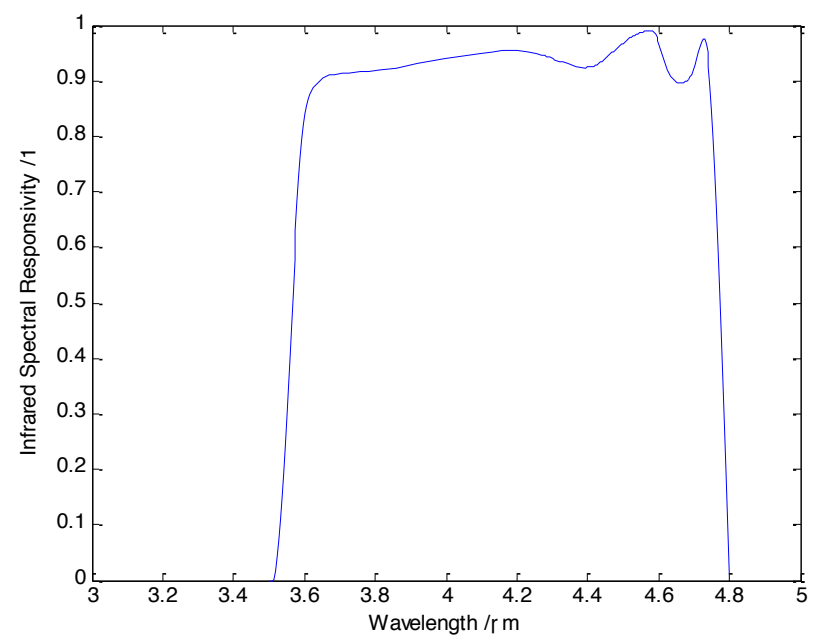

Fig. 5. Infrared Spectral Responsivity Curve

As illustrated in Fig. 5, the cutoff wavelength of the spectral response curve is approximately $3.5 \sim 4.8 \mu \mathrm{m}$. The overall spectral response within the wavelength range is greater than 0.9 , slightly undulating. The relevant materials [13], [14] show that the measurement error of spectral responsivity of infrared detector is generally less than $1.1 \%$.

From Eq.(17) and the known input value, the final error of $g_{H}$ is $1.1 \%$. Similarly, the final error of $g_{L}$ is $1.1 \%$. 


\subsection{Result of Radiance calibration coefficients aqucisition}

This article follows the principle, so as to ensure effective and reliable of the acquired data. Radiometric calibration data collected are shown in Table 4.

Table 4. Blackbody temperature and gray value

\begin{tabular}{c|c|c|c|c|c|c|c}
\hline$T(~)$ & 5 & 10 & 15 & 20 & 25 & 30 & 35 \\
\hline gray & 28036 & 32266 & 35839 & 40039 & 45023 & 48881 & 53625 \\
\hline \hline
\end{tabular}

On the basis of Eq.(6), the pupil radiance can be gotten by substituting the blackbody temperature, emissivity and the spectral response of infrared instrument, as follows:

Table 5. Pupil radiance and gray value

\begin{tabular}{c|c|c|c|c|c|c|c}
\hline $\begin{array}{c}L \times 10^{-5} \\
\left(\mathrm{Wcm}^{-2} \mathrm{sr}^{-1}\right)\end{array}$ & 0.479 & 0.592 & 0.725 & 0.883 & 1.068 & 1.284 & 1.5342 \\
\hline gray & 28036 & 32266 & 35839 & 40039 & 45023 & 48881 & 53625 \\
\hline \hline
\end{tabular}

In accordance with Table 5, the infrared radiation calibration data can be plotted as the blue line in Fig.6. Due to the linear region of the data in the MWIR band, the blue line can be linear fitted to the red line by using least square method.

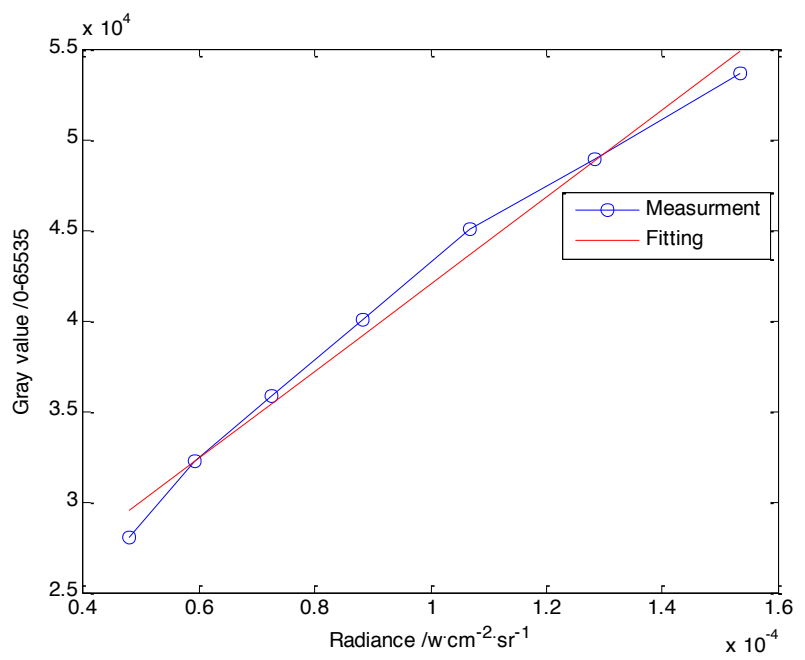

Fig. 6 Radiance calibration of infrared imaging system

By fitting, infrared camera radiometric calibration coefficient was gotten, $K=2.4 \times 10^{8}$. Since the fitting data did not completely overlap the measured data, the fitting error is introduced to $K$. Under the constraint of the linear fitting model and least square fitting method [15], the most appropriate and the optimized fitting result is uniquely determined, $\mathrm{RMSE}=1138$ and mean gray value is $\bar{g}=40530$. Taking RMSE values into Eq.(14), we obtain the relative standard deviation of radiance calibration coefficient $K$, $\sigma_{r}(K)=2.8 \%$. Take the data into Eq.(18), the final error is $3.01 \%$.
4.4 Result of MWIR average atmospheric transmittance

Blackbody temperature $T_{H}$ and $T_{L}$ are known from section 3.2 respectively: $T_{H}=35^{\circ} \mathrm{C}, T_{L}=15^{\circ} \mathrm{C}$. And the gray values of high and low temperature blackbody were obtained by extracting blackbody area from the images: $g_{H}=51107, g_{L}=34489$. Infrared radiance calibration coefficient was obtained from section 3.3, and $K=2.4 \times 10^{8}$. Thus the MWIR average atmospheric transmittance can be deduced by replace the input data referred by Eq.(7) with the values acquired in section 3 . And the result is $\tau=0.835$, which is in line with the empirical value of the sunny atmospheric transmittance.

\subsection{Error of MWIR average atmospheric transmittance} From the foregoing analysis, the errors of high and low temperature blackbody were: $\sigma_{r}\left(T_{H}\right)=0.87 \%, \sigma_{r}\left(T_{L}\right)=2.4 \%$. And the errors of infrared gray values are as follows: $\sigma_{r}\left(g_{H}\right)=1.1 \%$ and $\sigma_{r}\left(g_{L}\right)=1.1 \%$. Moreover, the radiance calibration coefficient error is $\sigma_{r}(K)=3.01 \%$. Instituting the above data into Eq.(10), the MWIR average atmospheric transmittance measurement error is calculated as $1.5 \%$, which is the integrated error of the entire measurement system and measure method, reflecting the effectiveness of the measurement of the MWIR average atmospheric transmittance.

\section{Conclusion}

This paper analyzes the mid-infrared atmospheric transmittance measurement principle, and acquires the various input data of atmospheric transmittance measurements required. Then, according to the measurement principle, the MWIR average atmospheric transmittance error model is theoretically derived and established to study the effects of each component of the error link to the entire error of atmospheric transmittance. In addition, through the specific data processing and error analyzing to the test, the measurement component and corresponding sub-errors are obtained. Ultimately, a comprehensive theoretical analysis and testing process are deducted to seek a mid-infrared atmospheric transmittance and the measuring error. The work of this paper shows that the measurement of atmospheric transmittance and error analysis reflect the primary factors of the measurement link. The results demonstrate that the measured values meet with the empirical atmospheric transmittance in sunny day very well. Moreover, the measurement error is less than $2 \%$, proving the validity of the measurement data from the perspective of the actual measurement.

\section{Acknowledgment}

The corresponding author of this paper is Wenhai $\mathrm{Xu}$, professor of Dalian Maritime University. And this work is financially supported by the Fundamental Research Funds for the Central Universities (Grant No. 017163, and Grant No. 3132013306).

\section{References}

1. Dario Cabib, "Complete affordable system for simultaneous VISNIR and MWIR/LWIR spectral atmospheric transmittance measurements
(ATMS)", Proceedings of the SPIE - Atmospheric Propagation VII 7685(76850), 2010, pp.1-10. 
Yuanyuan Ji, Wenhai Xu, Ying Li, Qilei Cao, Yukun Sun, Debin Ma and Ming Zhao/

Journal of Engineering Science and Technology Review 7 (1) (2014) 21 - 28

2. Da Mou, Quanyong Li, Jianing Dong, "Analysis on ground-based infrared detection system detecting the infrared magnitude of space targets", Infrared and Laser Engineering, 40(9), 2011, pp.1609-1613.

3. Zhangye Wang, "Study of the realistic infrared image synthesis for ground objects and the realistic fusion of multi-spectral images", Doctoral Dissertation, Zhejiang University, 2002.

4. Jun Zhu, Wenqing Liu, Yihuai Lu, Minguang Gao, "Research on radiance measurements of target and background based on FTIR", Infrared Technology, 26(01), 2004, pp.52-55.

5. Heli Wei, Xiuhong Chen, Jie Zhan, Ruizhong Rao, "Atmospheric correction in the measurement of infrared radiance", Journal Of Atmospheric and Environmental Optics, 2(6), 2007, pp.472-478.

6. D. Sadot, "A technique for active measurment of atmospheric transmittance using an imaging system: implementation at $10.6 \mu \mathrm{m}$ wavelength", Infrared Physics \& Technology, 36(7), 1995, pp.11051114.

7. Michael Y. Engel, "Quantitative evaluation of errors in remote measurements using a thermal imager", Proceedings of the SPIE - 7th Meeting in Israel on Optical Engineering, 1442(7), 1990, pp.298-307.

8. Ciyin Yang, Jianping Zhang, Lihua Cao, "Infrared radiation measurement based on real-time correction", J. Infrared Millim. Waves, 30(3), 2011, pp.284-288.

9. Yetai Fei, "Error theory and data processing", Beijing: Machinery Industry Press, 2010

10. F. A. Sowan, "Applications of infrared detectors", London: Mullard Limited, 1971.

11. John A. Jamieson, Raymond H. Mcfee, "Infrared physics and engineering", New York: Mcgraw-Hill Book Company, 1973.

12. D. Türler, "Laboratory procedures for using infrared thermography to validate heat transfer models", Insulation Materials: Testing and Applications, 3(1320), 1997, pp.1-23.

13. Philippe Chorier, "From visible to infrared, a new detector approach", Infrared Technology and Applications, 6206(01), 2006, pp.1-11.

14. Zhiqiang Lin, Xiaobin Zheng, Lei ZhangJi Wang, "Calibration of spectral responsivity of infrared detector", Opto-Electronic Engineering, 35(2), 2008, pp.118-122.

15. Teng Wu, Lingli Wu, "High-resolution scheme based on the undetermined coefficient method and its application", Journal of Engineering Science and Technology Reivew, 6(1), 2013, pp.119-123. 